\title{
La inteligencia emocional y su relación con las finanzas personales en docentes universitarios de Durango México
}

C.P.C. Blanca Bárbara Chávez Baca

blancachavezbaca@gmail.com https://orcid.org/0000-0002-2168-7628 Centro Educativo de Líderes Durango

Dr. Rosalío Tortolero Portugal chalioby@hotmail.com https://orcid.org/0000-0002-4526-7417 Centro Educativo de Líderes Durango

Dr. Ernesto Geovani Figueroa González geovanifigueroa@yahoo.es https://orcid.org/0000-0002-7900-9141 Centro Educativo de Líderes Durango

\section{Dr. José Gerardo Ignacio Gómez Romero} gerardoignaciog@yahoo.com.mx https://orcid.org/0000-0002-6322-6133 Centro Educativo de Líderes Durango

\section{RESUMEN}

La presente investigación tiene como objetivo general, determinar la correlación que existe entre la inteligencia emocional y las finanzas personales en docentes de una universidad privada de la ciudad de Durango México. Para la recopilación de la información se utilizó la técnica de la encuesta. El instrumento de medición aplicado en la investigación, se conforma por 39 reactivos en escala Likert (5, siempre, 4, casi siempre, 3 , algunas veces, 2 , casi nunca y 1 , nunca) distribuido en tres dimensiones para la variable dependiente finanzas personales y tres para la variable independiente inteligencia emocional, el cual se aplicó a 50 docentes de las diferentes carreras, en el mes de noviembre del año 2021. En la investigación se utilizó el diseño no experimental de nivel descriptivo, correlacional y de corte transversal, recopilando la información en un periodo específico. Se concluye en que existe evidencia para afirmar que la inteligencia emocional presenta una correlación positiva media con las finanzas personales, obteniendo un coeficiente de Rho de Spearman de .302 y que la dimensión reparación emocional, es la dimensión de la inteligencia emocional que más relación presenta con las finanzas personales.

Palabras clave: finanzas personales; inteligencia emocional; correlación. 


\title{
Emotional intelligence and its relationship with personal finances in university professors from Durango Mexico
}

\begin{abstract}
The general objective of this research is to determine the correlation that exists between personal finances and emotional intelligence in teachers of a private university in the city of Durango, Mexico. The survey technique was used to collect the information. The measurement instrument applied in the research is made up of 39 items on a Likert scale (5, always, 4, almost always, 3, sometimes, 2, almost never and 1, never) distributed in three dimensions for the dependent variable finances personal and three for the independent variable emotional intelligence, which was applied to 50 teachers of the different careers, in the month of November 2021. In the research, the non-experimental design of descriptive, correlational and cross-sectional level was used, collecting the information in a specific period. It is concluded that there is evidence to affirm that emotional intelligence presents a medium positive correlation with personal finances, obtaining a Spearman Rho coefficient of .302 and that the emotional repair dimension is the dimension of emotional intelligence that presents the most relationship with personal finances.
\end{abstract}

Keywords: personal finance; emotional intelligence; correlation.

Artículo recibido: 15 noviembre. 2021 Aceptado para publicación: 10 diciembre 2021 Correspondencia: wblancachavezbaca@gmail.com Conflictos de Interés: Ninguna que declarar 


\section{INTRODUCCIÓN}

Las finanzas, son el conjunto de actividades que, a través de la toma de decisiones, mueven, controlan, utilizan y administran dinero y otros recursos de valor. (Padilla, 2014). Por su parte, Ferrel y Geoffrey (2012), manifiestan que las finanzas hacen referencia a todas las actividades relacionadas con la obtención de dinero y su uso eficaz. Mientras que, para Bodie y Merton, (2003) las finanzas, estudian la manera en que los recursos escasos se asignan a través del tiempo. Por lo anterior, consideramos que finanzas sería la manera inteligente de distribuir el dinero a través de la vida productiva de la empresa, pero también de los individuos.

De acuerdo con Morales (2013), cuando se habla de finanzas personales, simplemente se hace referencia a la manera en que las personas se relacionan con el entorno mediante el uso del dinero. Las finanzas personales, son el uso que se le da al dinero, un buen uso de nuestro dinero lleva a incrementarlo y a poner en terreno firme nuestro porvenir financiero, para ello se requiere tener una buena inteligencia financiera, de manera tal, que podamos ir tomando buenas decisiones con respecto al dinero que ingresa a nuestras finanzas personales. Cada decisión que se toma cotidianamente, tiene que ver con nuestras finanzas y debe ser tomada de manera inteligente, llegando a considerar el nivel de inteligencia emocional como un factor clave para la buena gestión de las finanzas personales, sobre todo, a través de la vida productiva de las personas, de manera tal, que, a la edad de su retiro laboral, éstas puedan seguir disfrutando de su mismo nivel de vida obteniendo para ello ingresos pasivos distintos a su pensión.

Por su parte, Goleman (1995), define a la inteligencia emocional como la capacidad para reconocer los sentimientos propios y los sentimientos de los demás, de discriminar entre ellos y utilizar esa información para guiar nuestro pensamiento y nuestras acciones. En este sentido, las emociones pueden ser la mejor fortaleza o la mayor debilidad en el manejo del dinero, por ello, se aprecia a la inteligencia emocional como una buena herramienta para crear hábitos de ahorro, así como buenos hábitos de consumo, llevando una buena planeación financiera, estableciendo un presupuesto personal, que nos ayuda, a tener finanzas personales sanas, ya que al tomar decisiones es de suma importancia que nuestras emociones se encuentren controladas, para no dejarnos llevar por impulsos o incluso ser precavidos de más, a causa de nuestras emociones. 
De aquí surge el interés de llevar a cabo la presente investigación, la cual tiene como objetivo determinar la relación que existe entre la inteligencia emocional y las finanzas personales, de acuerdo a la perspectiva de los docentes de la Universidad Autónoma de Durango campus Alamedas, de la ciudad de Durango, México. Administrar el dinero propio, ahorrar, medir el gasto, controlar la deuda propia y en general realizar un presupuesto personal, son elementos que definen el manejo de las finanzas personales. En este sentido, Silva y Vargas (2020), manifiestan que un trabajador utiliza un aproximado de 20 horas de su horario laboral tratando de resolver sus problemas financieros, situación que evidentemente afecta el desempeño de las funciones designadas en horas laborales.

La educación financiera se encuentra en proceso de desarrollo y no está alineada con el plan de estudios de las instituciones educativas, se debe priorizar este tema e incluirlo de manera trasversal en las carreras universitarias, (Romero 2021). En este sentido, el Banco de Desarrollo de América Latina (2013) manifiesta, que la educación financiera puede empoderar a las personas al permitirles administrar de mejor manera sus recursos y las finanzas de sus familias.

Así mismo, Silva y Vargas (2020), señalan que debe existir un plan de educación financiera en los docentes universitarios que les permita brindar mejoras en la planificación de sus ingresos y en algunos casos mejorar su situación crediticia que permita poner en práctica el ahorro y de esta manera realizar una adecuada gestión, llevándolos a no tener problemas financieros al fin de mes.

El Banco Mundial de la Mujer (2008), brindó un manual que articula a la educación financiera en varias áreas, la principal de ellas es el presupuesto familiar o personal que funciona como principal herramienta de control de la economía doméstica, ya que es un instrumento de previsión y anticipación que se elabora a partir de los ingresos y de los gastos y a partir de ahí se pueden ir comprobando si las previsiones se cumplen, es decir si se está gestionando bien y alcanzando los objetivos propuestos. Además, el Banco Mundial de la Mujer (2008), propone realizar un presupuesto personal o familiar, se trata de realizar un cuadro de doble entrada, considerando aspectos tales como los ingresos y gastos, clasificando estos últimos como fijos, corrientes y ocasionales, dividiendo una columna para cada uno de ellos. 
Por otro lado, Salgado (2016), manifiesta la importancia que tiene controlar la situación crediticia para el manejo de las finanzas personales, es decir, es necesario analizar que las personas se encuentren al tanto de sus deudas para pagarlas en tiempo y forma. Por su parte, Borghino (2012), afirma que los gastos tienden a ser mayores que los ingresos y así es como se empieza a incorporar en la vida de las personas el síndrome del hámster, síndrome que aparece cuando se gasta en cosas que no producirán dinero, es decir, se adquieren bienes que no aumentarán su riqueza sino al contrario, González (2009) lo llama consumismo. En este sentido, Salgado (2016), señala que los hábitos de ahorro son factor clave para unas finanzas personales positivas, ya que el ahorro es el ingreso no gastado que tiene un propósito a futuro (De Gregorio 2012).

Con base en el análisis anterior de las finanzas personales y en aras de una correcta gestión por parte de los docentes universitarios, en sus finanzas personales, existen otros factores distintos a la educación financiera, que ayudan al buen manejo de éstas, llegando al final de mes sin problemas, practicando el ahorro e incluso la inversión. Dentro de esos factores hay uno relevante, denominado inteligencia emocional.

En este sentido, Tyson (2008) manifiesta que existen 10 problemas financieros personales comunes; 1). falta de planeación. 2). gastar de más. 3). comprar con un crédito de consumo. 4). demorar el ahorro para la jubilación. 5). caer presa de verborreas de ventas financieras 6). no hacer la tarea de comparar. 7). tomar decisiones basadas en los sentimientos. 8). no separar el grano de la paja. 9). exponerse a riesgos catastróficos y 10). enfocarse mucho en el dinero, centrándonos en el número siete, para analizar la variable inteligencia emocional en su relación con las finanzas personales. De acuerdo con Borghino (2012) la administración de las finanzas personales, no se trata sólo de inteligencia racional, las emociones, los afectos, los principios, los valores, también tienen un valor muy importante.

La inteligencia emocional es la habilidad de las personas para atender y percibir los sentimientos de forma apropiada y precisa, consiste en la capacidad de asimilar y comprender de manera adecuada los sentimientos para regular el estado de ánimo personal y de los demás. (Goleman 2010).

Fernández y Extremera (2002), señalan que en la inteligencia emocional se involucran cuatro grandes componentes; 1). percepción y expresión emocional, el cual consiste en reconocer de forma consciente las emociones propias e identificar qué se siente y ser 
capaz de darle una etiqueta verbal; 2). facilitación emocional, consiste en la capacidad para generar sentimientos que faciliten el pensamiento; 3). comprensión emocional, la cual implica integrar lo que se siente dentro del pensamiento propio y saber considerar la complejidad de los cambios emocionales y por último 4). regulación emocional, que consiste en dirigir y manejar las emociones tanto positivas como negativas de forma eficaz.

Estos cuatro grandes componentes están enlazados de forma escalonada, de manera que, para una adecuada regulación emocional es necesaria una comprensión emocional eficaz y para una buena comprensión se requiere una apropiada percepción emocional. Por lo que, la inteligencia emocional no es sinónimo de las habilidades que tenga la persona, tampoco del carácter, ni de su personalidad, tampoco es la suma de éstas, sino la fusión que convive en la persona de manera integrada.

Por su parte, Salovey y Meyer (1990), establecen criterios para medir la inteligencia emocional, separándolos en tres dimensiones; 1). atención emocional, la cual mide la capacidad de sentir y expresar sentimientos de manera adecuada. 2). claridad de sentimientos, que mide la capacidad de comprender de manera adecuado sus estados emocionales y 4). reparación emocional, la cual mide la capacidad de regular y gestionar estados emocionales adecuados a cada situación.

Finalmente, las decisiones y las acciones de las personas dependen tanto y en ocasiones más de sus propios sentimientos y pensamientos, por lo cual, la capacidad de los profesores para captar, comprender y regular sus emociones es el mejor índice del equilibrio emocional y se encontrará listo para tener una buena gestión de sus finanzas personales.

\section{ESTRATEGIAS METODOLÓGICAS O MATERIALES Y MÉTODOS}

La investigación es de tipo no experimental, ya que se realiza sin manipular las variables, es decir, no se modifica de forma intencional la variable independiente para determinar su efecto sobre la variable dependiente. Es transversal en cuanto a su temporalidad ya que se desarrolla en un momento específico y no se toman mediciones o captan resultados a lo largo del tiempo, es descriptiva porque muestra las características principales de la población objeto de estudio y es correlacional porque analiza cómo se presenta el grado de asociación entre las variables de estudio y determina la incidencia de una con otra. La recopilación de la información fue a través de la técnica de la encuesta, la cual se aplicó 
en el mes de noviembre del año 2021 a 50 docentes de una Universidad Privada de la Ciudad de Durango México.

El diseño del instrumento utilizado en la investigación para medir la variable dependiente fue propuesto por Silva-Díaz (2019). Por su parte, para medir la variable independiente se utilizó la escala propuesta por Salovey y Mayer (1990). El cuestionario está integrado por 39 reactivos en una escala Likert con cinco opciones que va de (5, siempre, 4 , casi siempre, 3 , algunas veces, 2 , casi nunca y 1 , nunca) distribuidos en las variables finanzas personales e inteligencia emocional. En lo que respecta a la variable dependiente que son las finanzas personales, incluye tres dimensiones (presupuesto personal, situación crediticia y hábitos de consumo). Por su parte, la variable independiente que corresponde a la inteligencia emocional incluye tres dimensiones (atención emocional, claridad de sentimientos y reparación emocional).

Para realizar la correlación, se utilizó el coeficiente de correlación Rho de Spearman, el cual es un método no paramétrico, comúnmente utilizado en las ciencias sociales. Este coeficiente es un instrumento para determinar la dependencia o independencia de dos variables aleatorias, el cual se cuantifica desde -1.0 hasta +1.0 (Mondragón, 2014).

De acuerdo al planteamiento metodológico descrito, las variables estudiadas establecen un modelo hipotetizado de estudio, el cual se representa en la siguiente figura:

Figura 1. Modelo Hipotetizado de las variables de estudio.

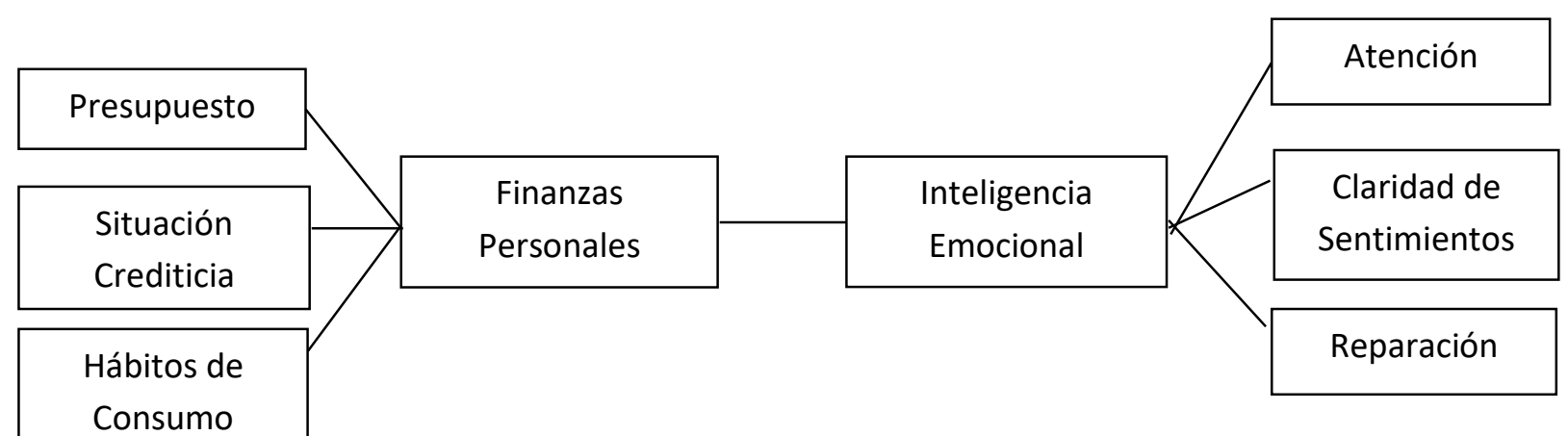

Fuente: Elaboración propia.

\section{RESULTADOS Y DISCUSIÓN}

El procesamiento de los datos se realizó a través del programa estadístico SPSS versión 26, donde la confiabilidad del instrumento de medición utilizado presenta un coeficiente de Alpha de Cronbach de $=.843$ y que según Hernández, Fernández y Baptista (2014), mientras más cercano a 1 se encuentre el Alpha de Cronbach tiene mayor confiabilidad. 
El diseño del instrumento que se aplicó en la investigación, comienza con los datos generales que incluye; edad, género, escolaridad y estado civil. En la tabla 1, se muestran los rangos de edad en los cuales se encuentran distribuidos los docentes encuestados. Se puede observar, que el 52\% se encuentra en el rango de 26 a 35 años representados por veintiséis docentes. El siguiente rango de edad que muestra una mayor frecuencia de docentes es el de 36 a 45 años con un $26 \%$. Cabe señalar que solamente un docente encuestado se encuentra en el rango menor a 25 años de edad, el $20 \%$ restante se encuentra por arriba de los 46 años.

Tabla 1.- Edad

\begin{tabular}{|c|c|c|c|c|c|}
\hline & & Frecuencia & Porcentaje & Porcentaje válido & $\begin{array}{l}\text { Porcentaje } \\
\text { acumulado }\end{array}$ \\
\hline \multirow{6}{*}{ Válido } & Menor de 25 años & 1 & 2.0 & 2.0 & 2.0 \\
\hline & 26 - 35 años & 26 & 52.0 & 52.0 & 54.0 \\
\hline & 36 - 45 años & 13 & 26.0 & 26.0 & 80.0 \\
\hline & 46 - 55 años & 5 & 10.0 & 10.0 & 90.0 \\
\hline & 56 años o más & 5 & 10.0 & 10.0 & 100.0 \\
\hline & Total & 50 & 100.0 & 100.0 & \\
\hline
\end{tabular}

Fuente: Información obtenida con el procesamiento de los datos en programa estadístico SPSS v.26.

Por su parte, la tabla 2, muestra una distribución equitativa de los docentes en relación al género. Se puede observar que el $54 \%$ de los docentes encuestados son del género femenino representados con 27 mujeres y el $46 \%$ son del género masculino, siendo 23 hombres.

Tabla 2.- Género

\begin{tabular}{|c|c|c|c|c|c|}
\hline & & Frecuencia & Porcentaje & Porcentaje válido & $\begin{array}{l}\text { Porcentaje } \\
\text { acumulado }\end{array}$ \\
\hline \multirow{3}{*}{ Válido } & Masculino & 23 & 46.0 & 46.0 & 46.0 \\
\hline & Femenino & 27 & 54.0 & 54.0 & 100.0 \\
\hline & Total & 50 & 100.0 & 100.0 & \\
\hline
\end{tabular}

Fuente: Información obtenida con el procesamiento de los datos en programa estadístico SPSS v.26.

La tabla 3, muestra la escolaridad de los docentes. Se puede observar que el $96 \%$ de los profesores encuestados cuentan con Licenciatura o Maestría en su formación profesional, 
representados por 24 docenes cada nivel. Así mismo, se muestra que solo un profesor cuenta con especialidad y uno con doctorado, sumando un $6 \%$ del total de encuestados.

Tabla 3.- Escolaridad

\begin{tabular}{|c|c|c|c|c|c|}
\hline & & Frecuencia & Porcentaje & Porcentaje válido & $\begin{array}{l}\text { Porcentaje } \\
\text { acumulado }\end{array}$ \\
\hline \multirow{5}{*}{ Válido } & Licenciatura & 24 & 48.0 & 48.0 & 48.0 \\
\hline & Especialidad & 1 & 2.0 & 2.0 & 50.0 \\
\hline & Maestría & 24 & 48.0 & 48.0 & 98.0 \\
\hline & Doctorado & 1 & 2.0 & 2.0 & 100.0 \\
\hline & Total & 50 & 100.0 & 100.0 & \\
\hline
\end{tabular}

Fuente: Información obtenida con el procesamiento de los datos en programa estadístico SPSS v.26.

En relación al estado civil, en la tabla 4 se puede observar que el $50 \%$ de los docentes son solteros representados por 25 profesores, seguidos por 19 docentes que se encuentran casados representando un $38 \%$ de la muestra. El resto de la distribución es mínima, donde solo uno se encuentra en unión libre y otro viudo. Así mismo, solo 4 docentes se encuentran divorciados.

Tabla 4.- Estado Civil

Frecuencia Porcentaje Porcentaje válido

Porcentaje acumulado

\begin{tabular}{|c|c|c|c|c|c|}
\hline \multirow{6}{*}{ Válido } & Soltero & 25 & 50.0 & 50.0 & 50.0 \\
\hline & Casado & 19 & 38.0 & 38.0 & 88.0 \\
\hline & Unión Libre & 1 & 2.0 & 2.0 & 90.0 \\
\hline & Divorciado & 4 & 8.0 & 8.0 & 98.0 \\
\hline & Viudo & 1 & 2.0 & 2.0 & 100.0 \\
\hline & Total & 50 & 100.0 & 100.0 & \\
\hline
\end{tabular}

Fuente: Información obtenida con el procesamiento de los datos en programa estadístico SPSS v.26.

Se realizó un análisis correlacional ya que el objetivo es establecer asociaciones entre dos variables, en este caso determinar si la variable inteligencia emocional tiene relación con las finanzas personales. 
Para cumplir con ello, se utiliza el coeficiente de correlación Rho de Spearman, el cual es una medida lineal que utiliza los rangos, números de orden, de cada grupo de sujetos y compara dichos rangos. (Mondragón 2014).

En la tabla 5, se muestra la correlación entre las variables analizadas en la presente investigación. Se puede observar, que la variable inteligencia emocional presenta un grado de asociación de =.302 con la variable finanzas personales, manifestándose una correlación positiva media según la clasificación que hace Mondragón (2014), en un estudio de intervención en fisioterapia, debido a que se encuentra en el rango de +0.11 a +0.50 .

Tabla 5.- Correlación entre las variables de estudio.

\begin{tabular}{|c|c|c|c|c|}
\hline & & & $\begin{array}{c}\text { Finanzas } \\
\text { Personales }\end{array}$ & $\begin{array}{c}\text { Inteligencia } \\
\text { Emocional }\end{array}$ \\
\hline \multirow{6}{*}{$\begin{array}{l}\text { Rho de } \\
\text { Spearman }\end{array}$} & \multirow{3}{*}{ Finanzas Personales } & $\begin{array}{l}\text { Coeficiente de } \\
\text { correlación }\end{array}$ & 1.000 & $.302^{*}$ \\
\hline & & Sig. (bilateral) & - & .033 \\
\hline & & $\mathrm{N}$ & 50 & 50 \\
\hline & Inteligencia & $\begin{array}{l}\text { Coeficiente de } \\
\text { correlación }\end{array}$ & $.302^{*}$ & 1.000 \\
\hline & \multirow[t]{2}{*}{ Emocional } & Sig. (bilateral) & .033 & . \\
\hline & & $\mathrm{N}$ & 50 & 50 \\
\hline
\end{tabular}

*. La correlación es significativa en el nivel 0,05 (bilateral).

Fuente: Información obtenida con el procesamiento de los datos en programa estadístico SPSS v.26.

Así mismo, la tabla 6, muestra las correlaciones de las dimensiones de la inteligencia emocional con las finanzas personales y se puede observar que la dimensión de reparación emocional, es la dimensión de la inteligencia emocional que presenta una correlación positiva media con las finanzas personales, obteniendo una rho de spearman de .416, encontrándose en un rango de +.051 a +0.75 según la clasificación que hace Mondragón (2014). 
Tabla 6.- Correlaciones de las dimensiones de la inteligencia emocional con las finanzas personales

Finanzas Atención Claridad de Reparación Personales Emocional Sentimientos Emocional

\begin{tabular}{|c|c|c|c|c|c|}
\hline \multirow{3}{*}{$\begin{array}{l}\text { Finanzas } \\
\text { Personales }\end{array}$} & $\begin{array}{l}\text { Correlación de } \\
\text { Pearson }\end{array}$ & 1 & .080 & .207 & $.416^{* *}$ \\
\hline & Sig. (bilateral) & & .581 & .148 & .003 \\
\hline & $\mathrm{N}$ & 50 & 50 & 50 & 50 \\
\hline \multirow{3}{*}{$\begin{array}{l}\text { Atención } \\
\text { Emocional }\end{array}$} & $\begin{array}{l}\text { Correlación de } \\
\text { Pearson }\end{array}$ & .080 & 1 & .176 & .233 \\
\hline & Sig. (bilateral) & .581 & & .222 & .104 \\
\hline & $\mathrm{N}$ & 50 & 50 & 50 & 50 \\
\hline \multirow{3}{*}{$\begin{array}{l}\text { Claridad de } \\
\text { Sentimientos }\end{array}$} & $\begin{array}{l}\text { Correlación de } \\
\text { Pearson }\end{array}$ & .207 & .176 & 1 & $.437^{* *}$ \\
\hline & Sig. (bilateral) & .148 & .222 & & .002 \\
\hline & $\mathrm{N}$ & 50 & 50 & 50 & 50 \\
\hline \multirow{3}{*}{$\begin{array}{l}\text { Reparación } \\
\text { Emocional }\end{array}$} & $\begin{array}{l}\text { Correlación de } \\
\text { Pearson }\end{array}$ & $.416^{* *}$ & .233 & $.437^{* *}$ & 1 \\
\hline & Sig. (bilateral) & .003 & .104 & .002 & \\
\hline & $\mathrm{N}$ & 50 & 50 & 50 & 50 \\
\hline
\end{tabular}

**. La correlación es significativa en el nivel 0,01 (bilateral).

Fuente: Información obtenida con el procesamiento de los datos en programa estadístico SPSS v.26.

\section{CONCLUSIÓN O CONSIDERACIONES FINALES}

Una vez realizada la investigación y haber examinado los resultados de la misma, se concluye que existe una relación significativa entre la inteligencia emocional y las finanzas personales en docentes universitarios de la Universidad Autónoma de Durango, Campus Alamedas, de la ciudad de Durango, Mex. Ya que, según los resultados planteados, la gestión que tiene cada docente de sus finanzas personales cambia de acuerdo al nivel de inteligencia emocional que se tenga. 
Se concluye también, que de las tres dimensiones analizadas de la inteligencia emocional, la de reparación emocional, contempla la relación más alta con las finanzas personales, toda vez que esta dimensión, mide la capacidad de regular y gestionar estados emocionales adecuados a cada situación, considerando que las finanzas personales son las actividades que, a través de la toma de decisiones, mueven, controlan, utilizan y administran dinero, quedando de manifiesto que al tomar decisiones financieras, influye considerablemente el estado de ánimo que se tenga en ese momento, por lo que si el docente se encuentra desmotivado o deprimido tomará decisiones distintas a que si estuviera motivado y con buen estado de ánimo, en cuanto al control, utilización y administración de su dinero.

Los resultados obtenidos en la presente investigación, servirán a la universidad objeto de estudio, tomar en cuenta, la importancia que tiene el nivel de inteligencia emocional de sus docentes para la buena o mala gestión que tengan de sus finanzas personales, y les permita tener una buena educación financiera. Además, valorar la implementación de planes de acción estratégicos que incentiven una buena gestión de las finanzas personales y un manejo de sus emociones, ya que, de lo contrario, pudiera afectar la productividad y calidad del desarrollo de su trabajo en las aulas, así mismo,

El estudio realizado también servirá para analizar el comportamiento de ambas variables en otras Instituciones educativas de nivel superior, quedando de manifiesto a la vez, la posibilidad de próximos estudios, donde se demuestre una relación significativa entre las finanzas personales y la productividad en docentes universitarios.

\section{LISTA DE REFERENCIAS}

Banco de Desarrollo de América Latina. (2013). La educación financiera en América Latina y el Caribe situación actual y perspectivas. (CAF, Ed.) Cyngular. https://www.oecd.org/daf/fin/financialeducation/OECD_CAF_Financial_Educat ion_Latin_AmericaES.pdf

Banco Mundial de la Mujer. (2008). Manual de educación financiera. Madrid, España: Fundación Laboral WWB en España. https://gref.org/nuevo/documentacion/manual_educacion_financiera.pdf

Bodie, Z. M. R., \& Merton, R. (2003). Finanzas, Editorial Prentice Hall.

Borghino, M. (2012). El arte de hacer dinero (El arte de). Grijalbo.

De Gregorio, J. (2012). Macroeconomía. Santiago de Chile: Pearson-Educación. 
Fernández-Berrocal P, Extremera-Pacheco N (2002) La Inteligencia Emocional como una habilidad esencial en la escuela. Revista Iberoamericana de educación, Universidad de Málaga, España.

Ferrel, O.C., \& Geoffrey, H.A. (2012). Introducción a los Negocios en un Mundo Cambiante. México. Editorial McGraw-Hill Interamericana, Séptima edición.

Goleman, Daniel. (1995) Inteligencia Emocional. Prime Edición al castellano. India. Editorial Kairos, S.A.

Goleman, Daniel (2010) Inteligencia Emocional. Primera edición digital. Editorial. India Kairos, S.A.

González, J. D. L. (2009). Consumo y consumismo. Algunos elementos trazan sobre estudiantes universitarios en México. Nómadas. Critical Journal of Social and Juridical Sciences, 21(1)

Hernández, R., Fernández, C., \& Bautista. (2014). Metodología de la Investigación (6th ed.). México, México: McGraw Hill.

Morales, V. M. (2013). Programa de Acondicionamiento Financiero. Tuxtla Gutiérrez, Chiapas: Escritores Noveles.

Mondragón, M. (2014). Uso de la correlación de Spearman en un estudio de intervención en fisioterapia. Movimiento Científico, 8(1), 98-104.

Padilla, V. M. (2014). Introducción a las Finanzas. México: Grupo Editorial Patria, S.A. de C.V.

Romero Miñano, M. C. (2021). La educación financiera desde la visión de docentes universitarios de ciencias económicas en Latinoamérica.

Salgado Obregón, A. J. (2016). Análisis de Finanzas personales y su efecto en el desarrollo socio-económico de socios y socias de la cooperativa de viviendas COVISCOF, RL Departamento de Estelí, I Semestre 2015 (Doctoral dissertation, Universidad Nacional Autónoma de Nicaragua, Managua).

Salovey, P. \& Mayer, J. D. (1990). Inteligencia Emocional. Imaginación, cognición y personalidad. Estados Unidos de América.

Silva Díaz, L. A., \& Vargas Vilca, L. O. (2020). La educación financiera para mejorar las finanzas personales de los docentes de la institución educativa N 00518 del distrito de Yantalo, 2019. 
La inteligencia emocional y su relación...

Tyson, E. (2008). Finanzas Personales Para Dummies (Quinta ed.). Estados Unidos de América: Wiley Publishing. 